\title{
Inhibition of microRNA-17/20a suppresses cell proliferation in gastric cancer by modulating UBE2C expression
}

\author{
YINGYI ZHANG $^{1 *}$, TING HAN $^{2 *}$, GUO WEI $^{2}$ and YAJIE WANG ${ }^{1}$ \\ Departments of ${ }^{1}$ Oncology and ${ }^{2}$ General Surgery, Changhai Hospital, Shanghai 200433, P.R. China
}

Received December 1, 2014; Accepted January 7, 2015

DOI: 10.3892/or.2015.3835

\begin{abstract}
RNAs (miRNAs) are small non-coding RNAs that potentially play a critical role in carcinogenesis. Increasing evidence indicates that the miR-17/20 cluster is upregulated in numerous types of human cancers including gastric cancer which suggests that the miR-17/20 cluster may play an important role in tumorigenesis. However, its role in gastric cancer carcinogenesis remains poorly defined due to the lack of target gene information. The aim of the present study was to investigate the target genes of the miR-17/20 cluster and their role in the tumor growth of gastric cancer. We found that both miR-17 and miR-20a (miR-17/20a) target the UBE2C gene in gastric cancer cells. Luciferase assay, qRT-PCR and western blot analysis confirmed that UBE2C is a direct target of miR-17/20a in gastric cancer cells. Our results showed that the expression of UBE2C was positively regulated by miR-17/20a at both the mRNA and protein levels. Moreover, miR-17/20a was upregulated and positively associated with UBE2C in the gastric cancer tissues when compared to the adjacent nontumor tissues. Inhibition of miR-17/20a in gastric cancer cells was statistically correlated with a decrease in cell growth. These results demonstrate that upregulation of miR-17/20a promotes gastric cancer cell growth by targeting UBE2C and inhibition of their levels is a potentially promising therapeutic strategy for gastric cancer.
\end{abstract}

Correspondence to: Professor Yajie Wang, Department of Oncology, Changhai Hospital, Shanguai 200433, P.R. China

E-mail: yajiewa0459@163.com

Professor Guo Wei, Department of General Surgery, Changhai Hospital, Shanguai 200433, P.R. China

E-mail: wgdr2004@163.com

${ }^{*}$ Contributed equally

Abbreviations: CDS, coding DNA sequence; UTR, untranslated region; mRNA, messenger RNA; qRT-PCR, quantitative real-time PCR; mRNA, messenger RNA; miRNA, microRNA; miR-17, microRNA-17; miR-20a, microRNA-20a; CLASH, crosslinking, ligation, and sequencing of hybrids; FBS, fetal bovine serum

Key words: gastric cancer, miR-17, miR-20a, UBE2C, proliferation

\section{Introduction}

Gastric cancer is one of the most common types of cancer and is the second most frequent cause of cancer-related death worldwide (1). In China, approximately two-thirds of patients diagnosed with gastric cancer develop unresectable disease, and more than half of patients present with recurrence after curative surgery. The median survival time for these patients is only 6-9 months (2). Much effort has been spent on the study of the biological mechanisms of gastric cancer, and a large number of tumor-suppressor genes and oncogenes have been reported in recent years $(3,4)$. However, the molecular mechanisms underlying the development of gastric carcinomas are still poorly understood.

microRNAs (miRNAs) are a class of highly conserved small non-coding RNAs that have important regulatory roles in animals by regulating gene expression post-transcriptionally (5). Many important cellular functions such as proliferation, differentiation, apoptosis, development and metabolism are known to be regulated by miRNAs (6). Up to one-third of human genes, including tumor-related genes, are predicted to be miRNA targets (7). High-throughput technologies such as microarrays and next generation sequencing have shown the global expression pattern of miRNAs, and several miRNAs which are dysregulated in several malignancies including gastric cancer may act as novel oncogenes or tumorsuppressor genes $(8,9)$. However, the mechanisms underlying their function in gastric cancer tumorigenesis remain unclear due to the lack of target gene information.

The miR-17/20 cluster, which is located on chromosome $13 q 31$, is expressed in many mammalian tissues. This cluster contributes to the development of the heart, lung, blood vessels and immune system (10-12). However, dysregulation of miR-17/20 has been reported in several types of cancers including breast cancer $(13,14)$, oral squamous cell carcinoma (15), lung cancer (16), lymphomas (17) and gastric cancer $(9,18)$. As dysregulation of miR-17/20 is related to a number of cancers, it has been hypothesized that miR-17/20 may play an important role in tumorigenesis and tumor development. However, the function of miR-17/20 particularly in gastric cancer remains unclear.

In the present study, we determined that UBE2C is a direct functional target of miR-17/20a in gastric cancer cells, and miR-17/20a were upregulated and positively associated with UBE2C in gastric cancer tissues compared to adjacent 
nontumor tissues. Furthermore, downregulation of miR-17/20a blocked tumor cell proliferation in vitro. These data suggest that the overexpression of miR-17/20a facilitates the development of gastric cancer.

\section{Materials and methods}

Specimens. Twenty-five gastric cancer tissues and adjacent nontumor tissues were obtained from the Department of General Surgery, Changhai Hospital (Shanghai, China) for RT-PCR analysis. All of the tissue samples were obtained from surgical removal and immediately snap frozen and stored in liquid nitrogen until use. The study protocol was approved by the Shanghai Changhai Hospital Ethics Committee. Informed consent was obtained from all patients.

Cell culture. Cell lines BGC-823, SGC-7901 and HEK 293T used in this study were purchased from the American Type Culture Collection (ATCC). BGC-823 and SGC-7901 were cultured and maintained in RPMI-1640 medium supplemented with $10 \%$ fetal bovine serum (FBS). HEK 293 T was cultured in Dulbecco's modified Eagle's medium (DMEM) containing $10 \%$ FBS. Cells were maintained at $37^{\circ} \mathrm{C}$ in a humidified atmosphere with $5 \% \mathrm{CO}_{2}$.

$R N A$ isolation and $q R T-P C R$. Total RNA was isolated from the gastric cancer tissues, adjacent nontumor tissues and gastric cancer cell lines using Trizol according to the manufacturer's instructions. Purified mRNA and miRNAs were detected by qRT-PCR assay using All-in-One miRNA qRT-PCR detection kit (GeneCopoeia, Rockville, MD, USA). All primers are listed in Table I. U6 small RNA was used as an internal control for normalization and quantification of miR-17/20a expression. $\beta$-actin was used as an internal control for normalization and quantification of UBE2C expression.

Luciferase assays. The luciferase reporter construct was constructed by cloning the human UBE2C mRNA sequence into the pMIR-Report construct (Ambion Inc., Austin, TX, USA). The wild-type or mutant UBE2C mRNA fragment was amplified and cloned into the luciferase reporter via SpeI and HindIII sites. All the primers are listed in Table I. Luciferase reporter assays were performed as follows. HEK $293 \mathrm{~T}$ cells plated in a 96 -well plate were co-transfected with $100 \mathrm{nM}$ single-stranded miRNA inhibitors or negative control oligonucleotides, $10 \mathrm{ng}$ of firefly luciferase reporter and $5 \mathrm{ng}$ of pRL-TK (Promega, Madison, WI, USA) using the INTERFERin ${ }^{\circledR}$ reagent (Polyplus-Transfection SA, Brant, France). Cells were collected $36 \mathrm{~h}$ after the last transfection and analyzed using the Dual-Luciferase Reporter assay system (Promega).

Oligonucleotide transfection. RNA oligos were chemically synthesized and purified by Genepharma Co. Ltd. (Shanghai, China). The sequence of the human miR-17 inhibitor was 5'-CUACCUGCACUGUAAGCACUUUG-3' and the human miR-20a inhibitor was 5'-CUACCUGCACUAUAAGCACU UUA-3'. Negative control oligonucleotides for the miRNA inhibitor were 5'-CAGUACUUUUGUGUAGUACAA-3'. The sequences of the UBE2C siRNAs were 5'-GAAGUACCU
Table I. All primers used in this study.

\begin{tabular}{ll}
\hline Name & \multicolumn{1}{c}{ Primer sequence $\left(5^{\prime} \rightarrow 3^{\prime}\right)$} \\
\hline miR-17 F & CAAAGTGCTTACAGTGCAGGTAG \\
miR-20a F & TAAAGTGCTTATAGTGCAGGTAG \\
U6 F & GTGCTCGCTTCGGCAGCAC \\
U6 R & AAAAATATGGAACGCTCACG \\
$\beta$-actin F & CAGCAAGCAGGAGTATGACG \\
$\beta$-actin R & GAAAGGGTGTAACGCAACTAA \\
UBE2C F & GGATTTCTGCCTTCCCTGAA \\
UBE2C R & GATAGCAGGGCGTGAGGAAC \\
UBE2C (WT) F & AAACTAGTAAGAAGTACCTGCAAGAAACC \\
UBE2C (WT) R & GGAAGCTTCCTGGCTGGTGACCTGC \\
UBE2C (MUT) F & AAACTAGTAAGAAGTTGGACGAAGAAACC \\
UBE2C (MUT) R & GGAAGCTTCCTGGCTGGTGACCTGC
\end{tabular}

F, forward primer; R, reverse primer; WT, wild-type; MUT, mutant.

GCAAGAAACCUACUCATT-3' (sense) and 5'-UGAGUA GGUUUCUUGCAGGUACUUCTT-3' (antisense) and for the control siRNAs were 5'-UUCUCC GAACGUGUCACGUTT-3' (sense) and 5'-ACGUGACACGUUCGUAGAATT-3' (antisense). The transfections were performed with INTERFERin reagent. The final concentration of miRNA inhibitors was $100 \mathrm{nM}$. The final concentration of siRNAs was $20 \mathrm{nM}$.

MTT assay. The in vitro growth of gastric cancer cells was measured using the MTT assay. Cells $(5,000)$ were seeded into each well of 96-well plates and transfected with miR-17/20a inhibitors or UBE2C siRNAs at a final concentration of 100 or $20 \mathrm{nM}$, respectively. On the day of harvest, $100 \mu \mathrm{l}$ of spent medium was replaced with an equal volume of fresh medium containing MTT $(0.5 \mathrm{mg} / \mathrm{ml})$. The plates were incubated at $37^{\circ} \mathrm{C}$ for $4 \mathrm{~h}$, and then the medium was replaced by $100 \mu \mathrm{l}$ of DMSO (Sigma, St. Louis, MO, USA) and plates were shaken at room temperature for $10 \mathrm{~min}$. The absorbance was measured at $570 \mathrm{~nm}$.

Immunoblotting. Total cellular extracts $(20 \mu \mathrm{g})$ were separated by a $4-20 \%$ Tris-glycine gel and then transferred to a PVDF membrane (Immobilon-P transfer membranes; Millipore Corp., Bedford, MA, USA). Following the transfer, the blots were blocked with $5 \%$ non-fat dry milk in PBS $+0.1 \%$ Tween-20 for $2 \mathrm{~h}$ and washed three times with PBS $+0.1 \%$ Tween-20 at $4^{\circ} \mathrm{C}$. The blots were then probed with a 1:200 dilution of the primary antibody against UBE2C (AB3935; Abcam, Cambridge, MA, USA), cyclin A (H-432; Santa Cruz Biotechnology, Santa Cruz, CA, USA) or $\beta$-actin (c-11; Santa Cruz Biotechnology). The blots were then probed with a 1:750 dilution of the secondary antibodies for $1 \mathrm{~h}$ at $4^{\circ} \mathrm{C}$, followed by washes in PBS $+0.1 \%$ Tween-20 and detection was carried out using an Odyssey Scanning system.

Statistical analysis. All statistical analyses were carried out using the SPSS 16.0 statistical software package. All data are presented as the mean \pm standard deviation. Student's t-test was used to analyze the difference between two experimental groups and a two-tailed $\mathrm{P}<0.05$ was taken to indicate statistical significance. 
Table II. List of genes that paired with miR-17 in the CLASH data.

Target genes

PLEKHM1, DAPK3, HEXIM1, KCTD7, ARIH1, TXLNA, RBM12B, ZNFX1, WASL, GNAS, PCGF5, ZBTB5, RALGAPA1, RALGAPA1, MLL, MAP3K9, KIF1A, TRIM71, ZNF598, MAP3K8, MKI67, ZIK1, NAP1L1,PPP1CA, RNF146, ZFYVE26, APP, APP, RUNDC1, TRIM8, BTN3A1, EIF4G2, FER, WAC, STIL, SUOX, FXR2, CPS1, GNB1, IRAK1, NUFIP2, C15orf41, CPSF1, MTF1, CCDC47, PDLIM5, ARHGAP10, BAZ2A, EHMT2, WEE1, ERLIN1, TMSB10, RPSA, LAS1L, MYCBP2, TUT1, RTCD1, TNFAIP1, PDPK1, PLAG1, TCEA2, AK1, RNF145, HDHD1A, PLS1, CCT6A, PBXIP1, TMEM19, PCNX, ELMO2, TRRAP, MOBKL1A, SLC25A28, HN1, ARPC2, NAT8L, PTTG1, TNPO3, ANKRD27, ILF3, LSM14A, LSM14A, LPIN1, GLO1, HSP90AA1, ATXN7, ERCC2, RTN3, SLC16A2, GANAB, TNFRSF10B, MTMR3, EFHC1, TOB1, RAB5B, RLF, MORF4L2, FANCA, HIST2H3A, ARHGEF7, CLPTM1, DCBLD2, PPP2R1A, BLVRA, ZNF507, KIAA1919, NBR1, DPYSL2, TUBB2C, C16orf63, PRPF8, GAPDH, KIAA1267, PGAM1, MT-ATP6, VPRBP, MNT, GPI, GDF11, POGZ, BACE1, TMTC4, MED13, EPB41L2, TRAP1, AC010619-1, DNMBP, CCND2, XIAP, SDHA, NOTCH2, EIF4G3, NONO, SMAD3, CHTF8, LAPTM4A, C5orf43, SON, TRIM44, CHST14, SEPT11, PRICKLE1, DCUN1D4, TRA2B, AMD1, FASN, C17orf42, SEPT2, GPM6A, EIF2C1, TSC2, NAGK, MED12, TMEM9, UQCRFS1, ABI2, EPB41L5, CTSA, RPL21, SURF4, ELP2, QARS, EIF2C1, SIPA1L3, ARHGAP5, MT-ATP6, ARL9, PER1, OFD1, FTH1, PLXNA1, PTBP1, ATRX, NAPEPLD, TAF9B, SH3GLB2, COX7B, WDR82, KIF5C, NUCKS1, LCOR, ZBED3, MDK, TMED10, TRUB1, DEPDC1, UBE3C, RBM5, HDAC10, AC010619-1, ASH1L, MFHAS1, DCTPP1, NFAT5, SLC25A37, FAM82B, UBE2C, CAP1, RPL7, EARS2, ENPP5, CANX, NOC2L, TMEM188, CBL, ATP5B, MT-CO2, EIF2C1, HIST2H4B, KDM4A, C6orf205, SLC25A3, CHD4, MRPS6, REEP5, RPL37, EIF2C1, CETN2, PELI1, MGEA5, COPS3, HIST2H2AA3, RYR2, TMEM90B, MYCBP2, OPTN, POGK, KAT2A, MTRF1L, EEF1 A1, PAIP1, PIK3CA, MT-ND2, HNRNPU, IGFBP5, MBNL1, MYC, CPE, C7orf44, RPS15A, AZIN1, APEX1, ZNFX1, HIST3H2A, HUWE1, HIST1H2AM, PSD3, TBC1D15, IMMT, HTT, ZNF689, RTTN, ASNS, MT-ND4, RAB23, ATF3, ACOT2, STK11, MT-ATP6, NPAS2, FAM8A1, MEN1, ADARB1, ZSWIM3, LARP1, RAB23, USP38, PPP1R15A, MT-ND4, PIGS, MRPL40, TRIM11, SOX4, TMEM165, GDAP1, HIST1H4C, RPS27A, DHX33, UBE4A, SLC35E2B, ZC3H18, TJP1, FBXO28, MOBKL1A

Newly published CLASH data in HEK 293 cells provided the direct evidence for miRNA:mRNA pairing [Helwak et al (19)].

Table III. List of genes that paired with miR-20a in the CLASH data.

Target genes

RBM12B, ZNFX1, ZNFX1, WAC, HEXIM1, LYPD6, LASS2, LEPREL4, LEPREL4, ZNF598, MAGOHB, EIF4G2, ARPC2, BNIP2, CCDC47, ZNF706, PHC1, NAP1L1, RBL2, SLC25A28, NETO2, NUDT21, ARFGEF2, PBXIP1, BTN3A1, RPS27, KLHL15, RUFY2, UQCRC1, KDM4D, CDC2L6, STIL, PPP2R1A, C9orf78, AKR7A2, PYGB, MT-ATP6, B4GALT2, YBX1, SELENBP1, RPL21, BAT2D1, ATL3, FOXJ3, MORF4L2, NCOA3, DCI, NUP188, MANEAL, EIF2C1, ADSS, PLXNA1, FLNA, AC004410-1, C20orf43, KLHL15, POGZ, CCNB1, WBP4, EIF2C1, CDK16, PPP2R1A, PTPRS, LDHB, GPN2, ARHGEF7, RFC3, BACH1, EIF2C4, MAN1C1, MRPL13, ABCA3, CTSA, ARL9, TMEM66, RPL18A, GATA6, STAT3, KIAA1191, USP10, CCDC88C, VEZF1, DLG5, TDRD3, TUBB, ORMDL3, PGK1, UBE2C, IL17RC, CEP120, ZNF398, XYLT2, BTBD2, PAQR5, NUP214, DLC1, MAD1L1, RNGTT, MLXIP, FBXO3, EIF2C1, EMR2, RBM10, LPHN3, C11orf59, TMX4, MRS2, RPL30, CDT1, L2HGDH, EIF2C1, PAIP1, CTR9, C11orf68, PTPN23, DPY19L4, TBC1D15, AP3D1, PSD3, NCOR2, TOMM20, SEPT2, RPL31, FBL, PPAN, C16orf68, PPP6R3, ZNF331, HAUS2, MPHOSPH8, DTX2, DDX5, KIF2C, PHYH, RPA2, MT-ATP6, TMEM97, IKZF5, C14orf135, AP3S2, PRKD3, APOA1BP, HMG20A, INTS3, GDI2, FHL3, KIAA0100, EIF2C1, COX5A, SSRP1, FGF7, UEVLD, ALDH18A1, RPS10, PLEKHM3, TCEA1, RTN2, INSIG1, PSMD2, ATP8B2, RPRD1A, CCDC75, PHF8

Newly published CLASH data in HEK 293 cells provided the direct evidence for miRNA:mRNA pairing [Helwak et al (19)].

\section{Results}

miR-17/20 a have a potential target site on UBE2C $m R N A$. The miR-17/20 cluster, which is located on chromosome $13 \mathrm{q} 31$, have a similar sequence and are hypothesized to have the same target genes. In order to investigate the biological significance and its underlying mechanisms of the dysregulation of miR-17/20a in gastric cancer, the potential target genes of miR-17/20a that function in gastric cancer pathogenesis were further analyzed. Newly published CLASH data in HEK 293 cells provided us with the direct evidence for miRNA:mRNA pairing (19). The CLASH data showed that both miR-17 and miR-20a targeted the coding DNA sequence (CDS) of UBE2C which is involved in many cellular processes including cell proliferation, migration, survival and other cellular functions (20). All genes targeted by miR-17 and miR-20a in the CLASH data are listed in Table II and Table III. After retrieving miR-17/20a potential binding sequences in the human, mouse, rat and horse, the alignments with miR-17 and miR-20a are illustrated in Fig. 1. Alignment results indicated that miR-17/20a may target the coding sequence of UBE2C using a $3^{\prime}$ end mode.

miR-17/20a directly target and upregulate UBE2C expression in gastric cancer cells. To determine whether miR-17/20a are capable of targeting and regulating UBE2C expression in gastric cancer cells, we performed the following experiments. Previous research has reported that miR-17/20a are upregulated in gastric cancer (9); thus, we performed a loss-of-function study. We created the luciferase reporter plasmid with the wild type or the mutant targeting sequence 


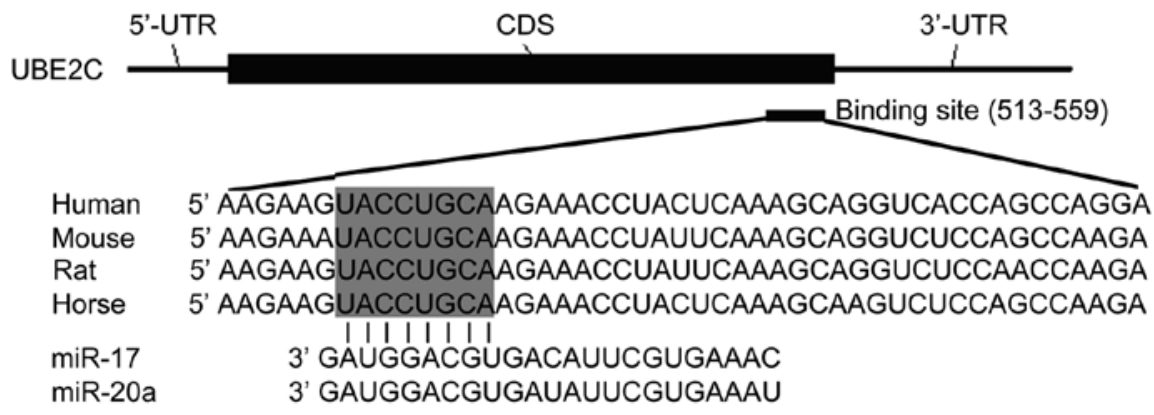

Figure 1. Bioinformatic analysis of the miR-17/20a binding site in UBE2C mRNA. Schematic diagrams of the UBE2C mRNA and the potential binding site for miR-17/20a. The upper panel shows one potential target site on CDS of UBE2C and the lower panel shows multiple sequence alignment of miR-17/20a with the binding site on CDS of UBE2C. Nucleotides in the dark-shaded boxes indicate sequences that are present in all four species. CDS, coding DNA sequence.

\section{A}

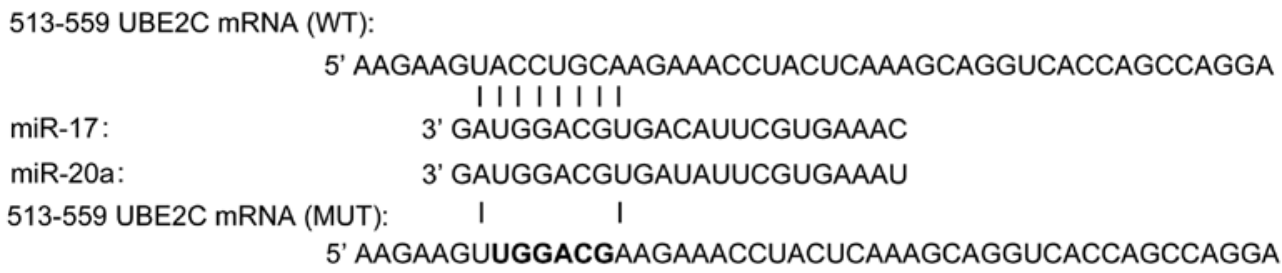

B

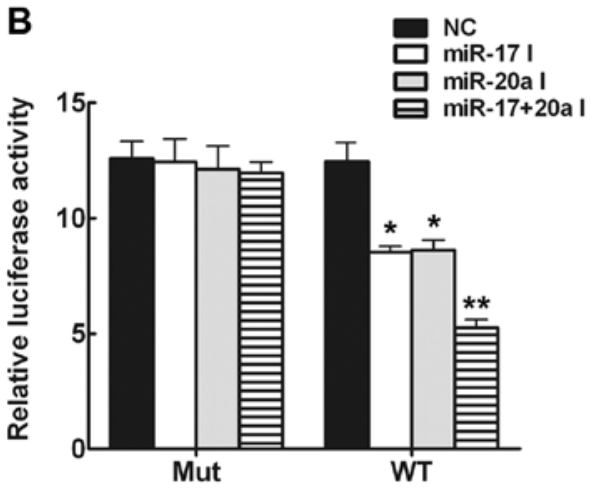

D

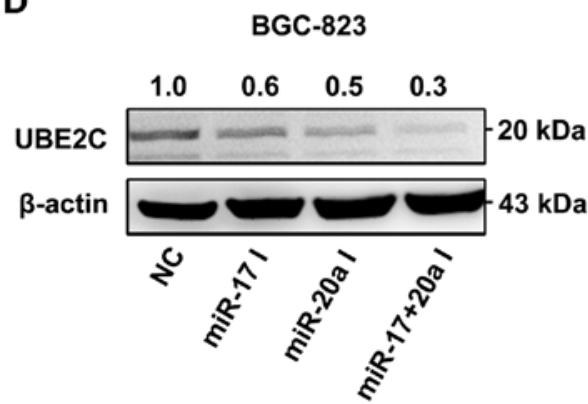

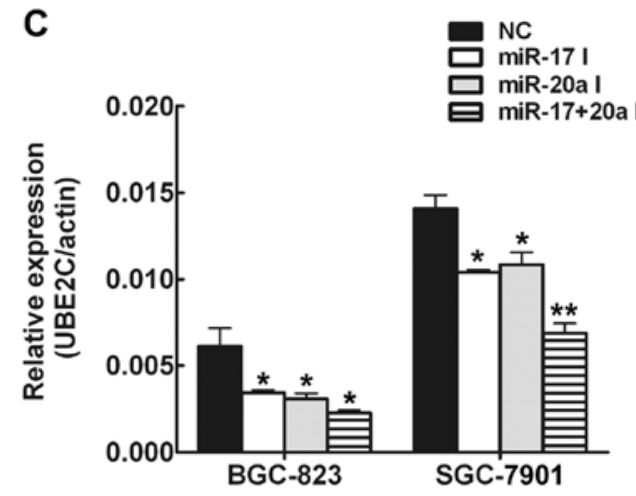

SGC-7901

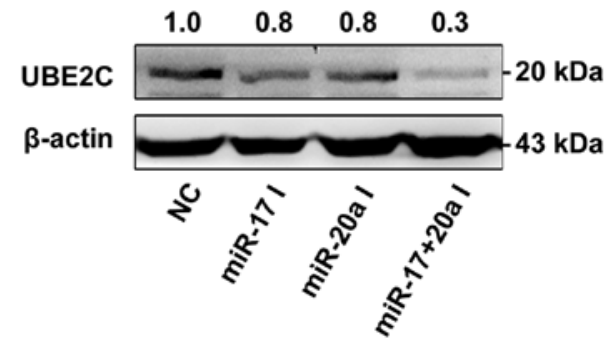

Figure 2. miR-17/20a target UBE2C in gastric cancer cells. (A) WT and Mut of putative miR-17/20a targeting sequences in UBE2C mRNA. Mutant sequences are shown in bold type. (B) Analysis of luciferase activity in HEK 293T cells. Cells were cotransfected with the firefly luciferase reporter plasmid containing putative miR-17/20a targeting sequences. Forty-eight hours after transfection, the cell lysates were assayed for luciferase activity and normalized to Renilla luciferase activity. The normalized luciferase activity of negative control oligonucleotides was set as relative luciferase activity 1 respectively. (C andD) Effects of miR-17/20a on the endogenous UBE2C expression levels. BGC-823 and SGC-7901 cells were cotransfected with miR-17 or miR-20a inhibitors and negative control oligonucleotides. Forty-eight hours after transfection, the cells were isolated, and the mRNA levels (C) and protein levels (D) of UBE2C were analyzed by qRT-PCR and WB, respectively ( $\mathrm{P}<0.05$ and ${ }^{* *} \mathrm{P}<0.01$, Student's t-test). WT, wild-type; Mut, mutant.

of UBE2C mRNA (Fig. 2A). The inhibitors of miR-17 and/ or miR-20 were transfected into HEK 293T cells. Luciferase assay was used to test the regulation of UBE2C by the
miRNAs. The results showed that inhibition of miR-17 or miR-20a decreased the luciferase activity, and inhibition of both miR-17 and miR-20a caused a more significant decrease 
A

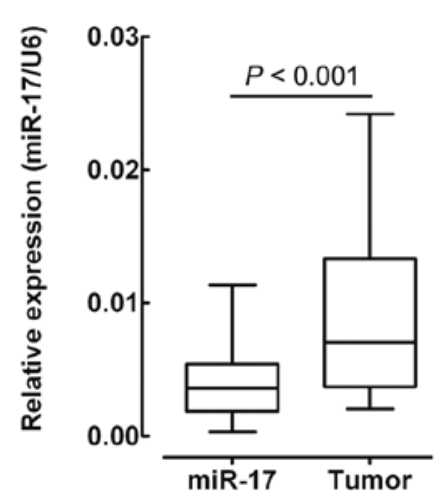

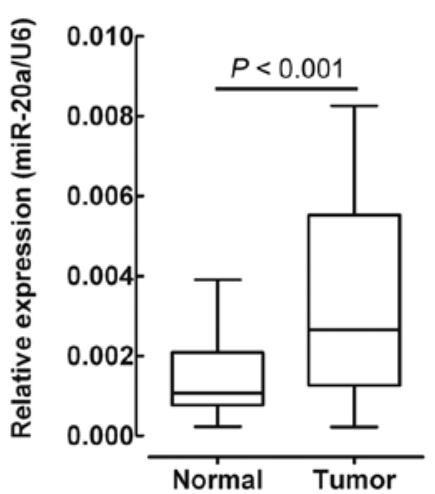

B

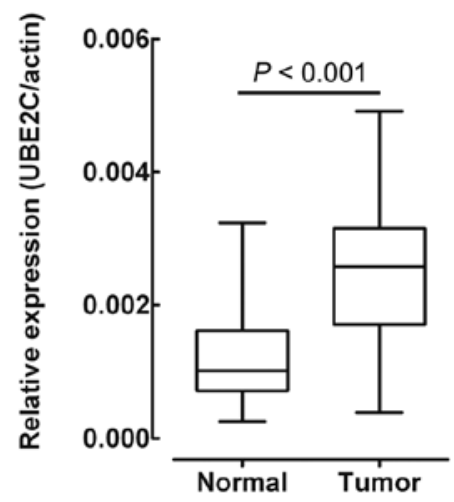

C
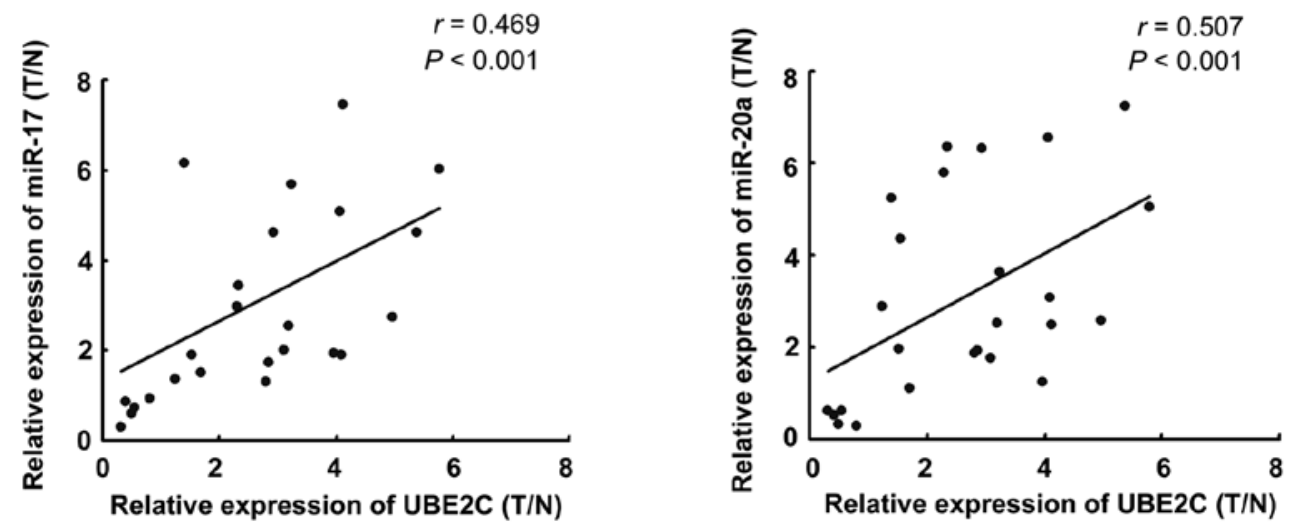

Figure 3. miR-17/20a are upregulated and are positively associated with UBE2C mRNA expression in gastric cancer samples. (A) qRT-PCR analysis of miR-17 and miR-20a expression in 25 pairs of gastric cancer tissues and their corresponding nontumor tissues. The expression of miR-17/20a was normalized to U6. (B) qRT-PCR analysis of UBE2C expression in the gastric cancer tissues and their corresponding nontumor tissues as indicated in A. The expression of UBE2C was normalized to $\beta$-actin. (C) A positive Spearman correlation between miR-17/20a and UBE2C mRNA levels was found in the 25 gastric cancer samples. T, tumor tissues; $\mathrm{N}$, adjacent nontumor tissues.

in luciferase activity, whereas the reporter plasmid with the mutant sequence of UBE2C produced no change in luciferase activity (Fig. 2A). These results revealed that miR-17/20a positively regulated UBE2C expression. Then, we explored whether the endogenous UBE2C in gastric cancer cells was regulated similarly. Two gastric cancer cells (BGC-823 and SGC-7901) were transfected with miR-17 and/or miR-20a inhibitors, and UBE2C mRNA and protein levels were examined by qRT-PCR and western blot analysis, respectively. The levels of UBE2C mRNA (Fig. 2B) and protein (Fig. 2C) were consistently and substantially downregulated by inhibition of miR-17 and miR-20a in the gastric cancer cells. A more significant decrease in UBE2C expression was also found in the cells which were transfected with both the miR-17 and miR-20a inhibitors. Our results indicated that miR-17/20a directly targeted UBE2C mRNA and positively regulated expression of UBE2C at both the mRNA and protein levels.

miR-17/20a are upregulated and positively associated with UBE2C mRNA levels in gastric cancer tissues. Previous research reported that miR-17/20a are upregulated in many cancers including gastric cancer (9) and overexpression of UBE2C is associated with worse patient outcomes in a number of cancers $(21,22)$. Our results demonstrated that UBE2C was positively regulated by $\mathrm{miR}-17 / 20 \mathrm{a}$ in gastric cancer cells. Next, we explored the correlation between miR-17/20a expression levels and the mRNA level of UBE2C in gastric cancer tissues. To determine the levels of $\mathrm{miR}-17 / 20 \mathrm{a}$ in gastric cancer samples, total RNAs were extracted from gastric cancer tissues, and the expression levels of miR-17/20a were analyzed using qRT-PCR and normalized to an endogenous control (U6 RNA). As shown in Fig. 3A, miR-17 and miR-20a were significantly increased in gastric cancer tissues vs. adjacent nontumor tissues (Fig. 3A), which agreed with a previous study (9). qRT-PCR assay was performed to detect the mRNA expression of UBE2C in the same gastric cancer samples and was normalized to $\beta$-actin. As shown in Fig. 3B, UBE2C mRNA levels were significantly increased in the gastric cancer tissues vs. the adjacent nontumor tissues, which was similar to previous findings in other cancers (21). After normalization to the expression value in normal tissues, RNA levels of miR-17/20a and mRNA levels of UBE2C in gastric cancer tissues were analyzed by Pearson's correlation coefficient analysis. Markedly, UBE2C mRNA levels were positively correlated with miR-17 ( $\mathrm{r}=0.469, \mathrm{P}<0.001)$ and miR-20a $(\mathrm{r}=0.507, \mathrm{P}<0.001)$ expression levels in gastric 
A

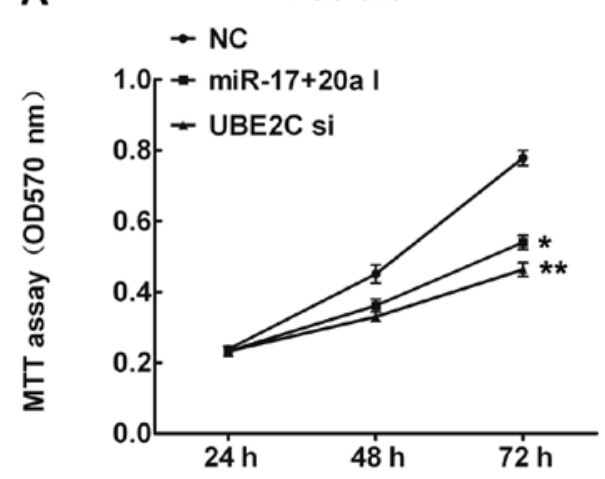

B

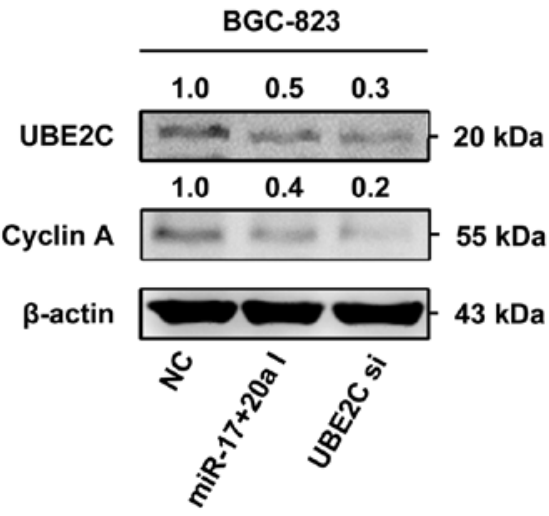

SGC-7901
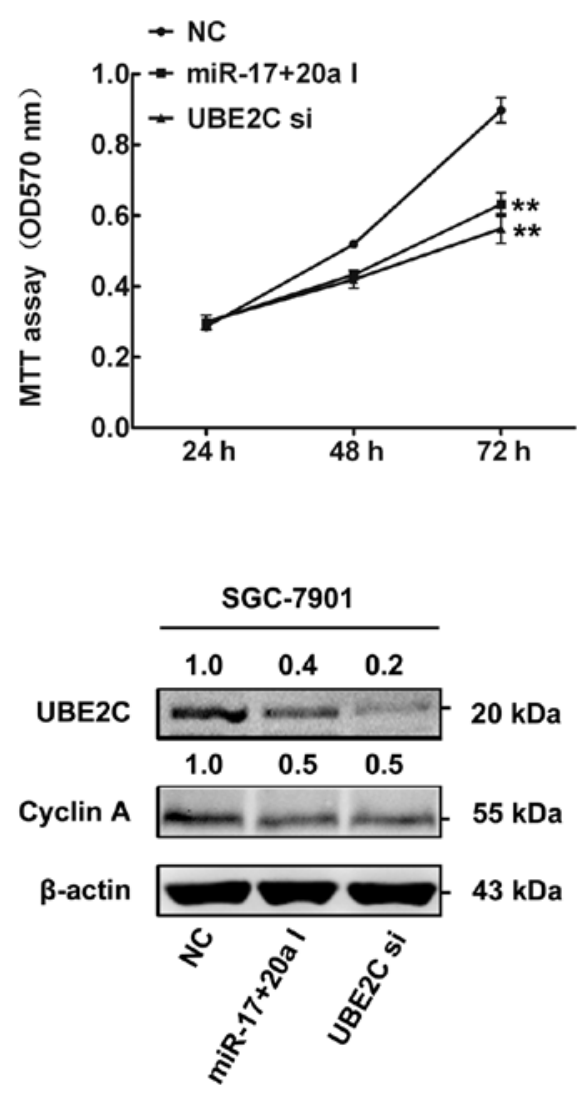

Figure 4. Downregulation of miR-17/20a inhibits proliferation of gastric cancer cells by targeting UBE2C. (A). The effects of UBE2C siRNA or miR-17/20a inhibitors on BGC-823 and SGC-7901 cell proliferation were determined by MTT assay at 24, 48 and $72 \mathrm{~h}$ after transfection. (B) Western blot analysis was performed to determine the expression levels of UBE2C and cyclin A after transfection of UBE2C siRNA or miR-17/20a inhibitors in the BGC-823 and SGC7901 cells $\left({ }^{*} \mathrm{P}<0.05\right.$ and ${ }^{* *} \mathrm{P}<0.01$, Student's t-test).

cancer tissues (Fig. 3C). Collectively, these results suggest that high endogenous miR-17/20a may target and upregulate the expression of UBE2C in gastric cancer.

Downregulation of miR-17/20a inhibits the proliferation of gastric cancer cells in vitro by targeting UBE2C. We then investigated the biological significance and its underlying mechanisms of the upregulation of miR-17/20a in gastric cancer. Cell proliferation is a key characteristic during tumorigenesis. However, the association of miR-17/20a with gastric cancer cell proliferation is unknown. Since overexpression of UBE2C increased the proliferation of cancer cells by modulating degradation of target genes such as cyclin A (23), and given that UBE2C is a direct target of miR-17/20a, we hypothesized that miR-17/20a may affect gastric cancer cell viability through UBE2C. In order to investigate this hypothesis, we performed the following experiments. Both miR-17 and miR-20a inhibitors or UBE2C siRNAs were transiently transfected into BGC-823 and SGC-7901 cells. In the cell proliferation assay, downregulation of miR-17 and miR-20a in BGC-823 and SGC-7901 cells resulted in significant suppression of cell proliferation which was similar to UBE2C siRNAs (Fig. 4A). Western blot analysis results determined that transfection of miR-17/20a inhibitors or UBE2C siRNAs decreased the expression of UBE2C and its downstream gene cyclin A in BGC-823 and SGC-7901 cells (Fig. 4B). Our results demonstrated that downregulation of UBE2C expression by miR-17/20a contributes, at least in part, to the suppression of the growth of gastric cancer cells.

\section{Discussion}

Recently, attention has been focused on the role of miRNAs in oncogenesis. In the present study, we focused on miR-17/20a which are increased in several tumor types including gastric cancer. Our results showed that UBE2C was one direct functional target of miR-17/20a in the gastric cancer cells. Upregulation of miR-17/20a was positively associated with UBE2C in gastric cancer tissues compared to adjacent nontumor tissues. Furthermore, inhibition of miR-17/20a blocked gastric cancer cell proliferation in vitro by downregulating UBE2C expression.

Recent discoveries have shown that miRNA, which is involved in almost every step of gastric carcinogenesis, has broad prospective application in the diagnosis and therapy of gastric carcinoma (24). Several miRNAs have been found to be downregulated in gastric cancer, such as miR-143, miR-145, miR-141, miR-31 and miR-106a, whereas some oncogenic miRNAs, such as miR-17, miR-20a, miR-21 and miR-27a, are upregulated (9). The human miR-17/20 cluster, located on chromosome 13q31, is dysregulated in several different cancers including gastric cancer. miR-17/20 inhibits cell growth via suppression of E2F1 expression (25). In mice, 
transgenic mice overexpressing miR-17 alone showed overall tissue growth retardation, smaller organs, and greatly reduced hematopoietic cell lineages (26). miR-17/20 inhibit cellular invasion and tumor metastasis in breast cancer by heterotypic signaling (14). The miR-17/20 cluster was found to inhibit the proliferation of mouse embryonic cardiomyocytes by targeting FOG-2 (12). miR-17/20a inhibited oral squamous cell carcinoma cell migration by regulating ITG $\beta 8$ (15). miR17/20a modulate macrophage inflammatory responses through targeting signal-regulatory protein $\alpha$ (11). However, the role of miR-17/20a in cancers particularly in gastric cancer remains unclear. In the present study, we confirmed that the oncogene UBE2C was directly targeted by miR-17/20a in gastric cancer. Surprisingly, we found that miR-17/20a positively regulated the expression of UBE2C at the mRNA and protein levels in gastric cancer cells. Moreover, upregulation of miR-17/20a was found to be positively associated with increased UBE2C expression in gastric cancer tissues, which suggests that UBE2C is positively regulated by miR-17/20a in human gastric cancer. Our results were similar to previous studies which showed that miRNA could positive regulate the expression of target genes $(27,28)$. However, the underlying mechanism of the positive regulation of UBE2C by miR-17/20a in gastric cancer remains to be explore.

UBE2C, located on chromosome $20 \mathrm{q} 13$, belongs to the E2 gene family and codes for a $19.6-\mathrm{kDa}$ protein involved in ubiquitin-dependent proteolysis. Overexpression of UBE2C causes chromosome mis-segregation and alters the cell cycle profile, which facilitates cell proliferation. UBE2C has been reported to be highly expressed in various types of cancers including ovarian carcinoma, metastatic prostate carcinoma, thyroid anaplastic carcinoma, hepatocellular carcinoma, and breast cancer (21,29-32). Knockdown of UBE2C expression by RNAi demonstrated the carcinogenetic role of UBE2C in proliferation, migration, and survival in cancer cell activities (33). However, the expression of UBE2C is largely unknown in human cancers particularly in gastric cancer. In the present study, we first report that UBE2C mRNA is highly expressed in gastric cancer tissues compared to matched adjacent nontumor tissues. To investigate the underlying mechanism of upregulation of UBE2C in gastric cancer. miRNA-binding site analysis revealed that UBE2C was one direct functional targets of miR-17/20a in gastric cancer cells. Inhibition of miR-17/20a in gastric cancer cells led to a significant UBE2C decrease and inhibition of cellular proliferation which was similar to the results of silencing UBE2C expression by siRNAs. It has also been shown that depletion of UBE2C suppresses anchorage-independent growth of breast cancer cells (34). Wagner and co-workers showed that selective silencing of UBE2C by siRNAs, in combination with TRAIL/DR5 agonistic antibodies, led to increased cell death in neoplastic but not nonmalignant human cells (21). In the present study, we demonstrated that miRNA-mediated knockdown of UBE2C inhibited the growth of gastric cancer cells, suggesting that targeting $\mathrm{UBE} 2 \mathrm{C}$ is a potential therapeutic strategy for gastric cancer.

Taken together, we demonstrated that miR-17/20a are upregulated in gastric cancer tissues. Inhibition of miR-17/20a directly downregulates UBE2C and inhibits gastric cancer cell proliferation. These data indicate that miR-17/20a-mediated knockdown of UBE2C may serve as a potential therapeutic strategy for gastric cancer.

\section{References}

1. Jemal A, Bray F, Center MM, Ferlay J, Ward E and Forman D: Global cancer statistics. CA Cancer J Clin 61: 69-90, 2011.

2. Yang L: Incidence and mortality of gastric cancer in China. World J Gastroenterol 12: 17-20, 2006.

3. Catalano V, Labianca R, Beretta GD, Gatta G, de Braud F and Van Cutsem E: Gastric cancer. Crit Rev Oncol Hematol 71: 127-164, 2009

4. Tamura G: Genetic and epigenetic alterations of tumor suppressor and tumor-related genes in gastric cancer. Histol Histopathol 17: 323-329, 2002.

5. Fabian MR, Sonenberg N and Filipowicz W: Regulation of mRNA translation and stability by microRNAs. Annu Rev Biochem 79: 351-379, 2010.

6. Bartel DP: MicroRNAs: genomics, biogenesis, mechanism, and function. Cell 116: 281-297, 2004.

7. Stefani G and Slack FJ: Small non-coding RNAs in animal development. Nat Rev Mol Cell Biol 9: 219-230, 2008.

8. Visone R and Croce CM: MiRNAs and cancer. Am J Pathol 174: 1131-1138, 2009.

9. $\mathrm{Wu} \mathrm{WK}$, Lee $\mathrm{CW}$, Cho $\mathrm{CH}$, et al: MicroRNA dysregulation in gastric cancer: a new player enters the game. Oncogene 29: 5761-5771, 2010.

10. Eiriksdottir G, Johannesdottir G, Ingvarsson S, et al: Mapping loss of heterozygosity at chromosome $13 \mathrm{q}$ : loss at $13 \mathrm{q} 12$-q13 is associated with breast tumour progression and poor prognosis. Eur J Cancer 34: 2076-2081, 1998.

11. Zhu D, Pan C, Li L, et al: MicroRNA-17/20a/106a modulate macrophage inflammatory responses through targeting signal-regulatory protein $\alpha$. J Allergy Clin Immunol 132: 426-436, 2012.

12. Xiang R, Lei H, Chen M, et al: The miR-17-92 cluster regulates FOG-2 expression and inhibits proliferation of mouse embryonic cardiomyocytes. Braz J Med Biol Res 45: 131-138, 2012.

13. Yu Z, Wang C, Wang M, et al: A cyclin D1/microRNA 17/20 regulatory feedback loop in control of breast cancer cell proliferation. J Cell Biol 182: 509-517, 2008.

14. Yu Z, Willmarth NE, Zhou J, et al: microRNA 17/20 inhibits cellular invasion and tumor metastasis in breast cancer by heterotypic signaling. Proc Natl Acad Sci USA 107: 8231-8236, 2010.

15. Chang CC, Yang YJ, Li YJ, et al: MicroRNA-17/20a functions to inhibit cell migration and can be used a prognostic marker in oral squamous cell carcinoma. Oral Oncol 49: 923-931, 2013.

16. Hayashita Y, Osada H, Tatematsu Y, et al: A polycistronic microRNA cluster, miR-17-92, is overexpressed in human lung cancers and enhances cell proliferation. Cancer Res 65: 9628-9632, 2005.

17. He L, Thomson JM, Hemann MT, et al: A microRNA polycistron as a potential human oncogene. Nature 435: 828-833, 2005.

18. Yao Y, Suo AL, Li ZF, et al: MicroRNA profiling of human gastric cancer. Mol Med Rep 2: 963-970, 2009.

19. Helwak A, Kudla G, Dudnakova T and Tollervey D: Mapping the human miRNA interactome by CLASH reveals frequent noncanonical binding. Cell 153: 654-665, 2013.

20. van Ree JH, Jeganathan KB, Malureanu L and van Deursen JM: Overexpression of the E2 ubiquitin-conjugating enzyme UbcH10 causes chromosome missegregation and tumor formation. J Cell Biol 188: 83-100, 2010.

21. Wagner KW, Sapinoso LM, El-Rifai W, et al: Overexpression, genomic amplification and therapeutic potential of inhibiting the UbcH10 ubiquitin conjugase in human carcinomas of diverse anatomic origin. Oncogene 23: 6621-6629, 2004

22. Okamoto Y, Ozaki T, Miyazaki K, Aoyama M, Miyazaki M and Nakagawara A: UbcH10 is the cancer-related E2 ubiquitinconjugating enzyme. Cancer Res 63: 4167-4173, 2003.

23. Rape M and Kirschner MW: Autonomous regulation of the anaphase-promoting complex couples mitosis to S-phase entry. Nature 432: 588-595, 2004.

24. Link A, Kupcinskas J, Wex T and Malfertheiner P: Macro-role of microRNA in gastric cancer. Dig Dis 30: 255-267, 2012.

25. O'Donnell KA, Wentzel EA, Zeller KI, Dang CV and Mendell JT: c-Myc-regulated microRNAs modulate E2F1 expression. Nature 435: 839-843, 2005. 
26. Shan SW, Lee DY, Deng Z, et al: MicroRNA MiR-17 retards tissue growth and represses fibronectin expression. Nat Cell Biol 11: 1031-1038, 2009.

27. Ma F, Liu X, Li D, et al: MicroRNA-4661 upregulates IL-10 expression in TLR-triggered macrophages by antagonizing RNA-binding protein tristetraprolin-mediated IL-10 mRNA degradation. J Immunol 184: 6053-6059, 2012.

28. Hussain M, Torres S, Schnettler E, et al: West Nile virus encodes a microRNA-like small RNA in the 3' untranslated region which upregulates GATA4 mRNA and facilitates virus replication in mosquito cells. Nucleic Acids Res 40: 2210-2223, 2012.

29. Chou CP, Huang NC, Jhuang SJ, et al: Ubiquitin-conjugating enzyme UBE2C is highly expressed in breast microcalcification lesions. PLoS One 9: e93934, 2014.

30. Welsh JB, Zarrinkar PP, Sapinoso LM, et al: Analysis of gene expression profiles in normal and neoplastic ovarian tissue samples identifies candidate molecular markers of epithelial ovarian cancer. Proc Natl Acad Sci USA 98: 1176-1181, 2001.
31. LaTulippe E, Satagopan J, Smith A, et al: Comprehensive gene expression analysis of prostate cancer reveals distinct transcriptional programs associated with metastatic disease. Cancer Res 62: 4499-4506, 2002.

32. Pallante P, Berlingieri MT, Troncone G, et al: UbcH10 overexpression may represent a marker of anaplastic thyroid carcinomas. Br J Cancer 93: 464-471, 2005.

33. Jiang L, Bao Y, Luo C, et al: Knockdown of ubiquitin-conjugating enzyme E2C/UbcH10 expression by RNA interference inhibits glioma cell proliferation and enhances cell apoptosis in vitro. J Cancer Res Clin Oncol 136: 211-217, 2010.

34. Fujita T, Ikeda H, Kawasaki K, et al: Clinicopathological relevance of UbcH10 in breast cancer. Cancer Sci 100: 238-248, 2009. 